\title{
Propriedades químicas de um Argissolo tratado sucessivamente com composto de lixo urbano
}

\author{
Chemical properties of an Ultisol successively treated with municipal waste compost
}

\author{
Alexandre Diehl Krob ${ }^{\mathrm{I}}$ Sílvio Paulo Moraes"II Pedro Alberto Selbach ${ }^{\mathrm{II}}$ \\ Fátima Menezes Bento ${ }^{\mathrm{III}}$ Flávio Anastácio de Oliveira Camargo ${ }^{\mathrm{I}^{*}}$
}

\section{RESUMO}

\begin{abstract}
Com o objetivo de avaliar as alterações em algumas propriedades químicas do solo sob adição sucessiva de composto de lixo urbano, conduziu-se um experimento a campo, em um solo Argissolo Vermelho de textura média na Estação Experimental Agronômica da UFRGS. Os tratamentos constaram de cinco doses de composto $(0,20,40,80$ e 160t $\left.\mathrm{ha}^{-1}\right)$, de um tratamento com adubo mineral e uma testemunha (Calagem e NPK), com aplicações sucessivas por um período de quatro anos. A adição de composto no solo aumentou o $\mathrm{pH}, \mathrm{CTC}, \mathrm{C}$ orgânico, $\mathrm{N}$ total, $\mathrm{P}$ e $\mathrm{Na}$ extraíveis, bem como da relação de adsorção de sódio (RAS), Ca e Mg trocáveis e o teor de $K$ extraível após a terceira aplicação. $O$ Al trocável teve os seus teores diminuídos após aplicações sucessivas de composto. $O s$ resultados permitiram concluir que aplicação de até $80 t$ ha $\mathrm{a}^{-1}$ por ano de composto de lixo urbano pode ser considerada como adequada para melhorar e, ou, manter as propriedades químicas do solo.
\end{abstract}

Palavras-chave: $p H, C T C, C$ orgânico, $N$ total, disponibilidade de nutrientes.

\section{ABSTRACT}

In order to assess the changes in some chemical properties of soil under the successive addition of urban waste compost, it was conducted a field experiment in an Ultisol soil at Agronomic Experimental Station of UFRGS. The treatments consisted of five successive applications of different doses of municipal solid waste compost $\left(0,20,40,80\right.$ and $160 t$ ha $\left.^{-1}\right)$ for a period of four years and a comparative treatment with mineral fertilizer and a control (without compost and fertilization). The addition of the compost increased soil pH, CEC, organic $C$, total $N$, extractable $P$ and $N a$ and the sodium adsorption ratio (SAR), calcium, and magnesium and extractable $K$ after the third application. The exchangeable Al had their levels reduced by successive applications of compost. The results showed that application of up to $80 \mathrm{tha}^{-1} \mathrm{yr}^{-1}$ of urban waste compost can be the dose recommended to improve and maintain the chemical properties of soil.

Key words: $p H, C E C$, organic $C$, total $N$, nutrient availability.

\section{INTRODUÇÃO}

A compostagem é um sistema bastante conhecido para a rápida estabilização e humificação de resíduos sólidos, bem como uma alternativa ambiental e economicamente vantajosa para o tratamento de resíduos sólidos como o lixo urbano (WEI et al., 2007). A utilização agrícola do composto de lixo urbano permite a ciclagem da matéria orgânica e de nutrientes e, consequentemente, a manutenção ou recuperação das propriedades químicas, físicas e biológicas dos solos (HARGREAVES et al., 2008).

Em termos de propriedades químicas, diversos trabalhos têm abordado alterações e melhorias na fertilidade do solo após a adição do composto de lixo. Essas melhorias referem-se, principalmente, a variações no $\mathrm{pH}$, aumento na capacidade de troca de cátions (CTC), na disponibilidade de nutrientes, na concentração de sais solúveis, etc. (MANTOVANI et

'ONG Curicaca, Porto Alegre, RS, Brasil.

"Departamento de Solos, Faculdade de Agronomia, Universidade Federal do Rio Grande do Sul (UFRGS), Av. Bento Gonçalves, 7712, 91540-000, Porto Alegre, RS, Brasil. E-mail: fcamargo@ufrgs.br. *Autor para correspondência.

II'Departamento de Microbiologia, Instituto de Ciências Básicas da Saúde, UFRGS, Porto Alegre, RS, Brasil. 
al., 2005; ABREU JUNIOR et al., 2001). Entretanto, para solos sob clima tropical, aplicações pontuais de composto de lixo urbano no solo não têm apresentado acréscimos significativos na CTC e somente aplicações consecutivas de doses de composto de lixo poderão aumentar a CTC desses solos (ABREU JUNIOR et al., 2001).

Os trabalhos que visam a avaliar a melhora nas propriedades químicas do solo pela adição de composto de lixo urbano são restritos a aplicações únicas e experimentos de vasos em casa de vegetação, sendo insuficientes para que se possam realizar recomendações seguras sobre a aplicação (ESCOSTEGUY et al., 1993). Todavia, aplicações sucessivas de composto de lixo urbano em doses mais elevadas podem causar efeitos negativos ao solo e às plantas, como aumento da salinidade, acúmulo de metais, lixiviação de nitratos e dispersão de argilas (ABREU JUNIOR et al., 2000). Desse modo, são necessárias informações de campo a respeito do efeito que aplicações sucessivas e doses elevadas de composto de lixo urbano podem causar nas propriedades químicas do solo. Com base nessas considerações, o presente trabalho teve como objetivo avaliar as alterações nas propriedades químicas de um Argissolo Vermelho de textura média que recebeu, durante cinco aplicações sucessivas, diferentes doses de composto de lixo domiciliar.

\section{MATERIAL E MÉTODOS}

O experimento foi conduzido em campo em um solo Argissolo Vermelho de textura média, na
Estação Experimental Agronômica da Universidade Federal do Rio Grande do Sul durante quatro anos. O solo, antes da instalação do experimento, foi analisado de acordo com TEDESCO et al., (1995) e apresentou os seguintes resultados: $\mathrm{pH}$ de 5,2; índice SMP de 6,2; $14 \mathrm{mg} \mathrm{kg}^{-1}$ de P; $181 \mathrm{mg} \mathrm{kg}^{-1}$ de K; $24 \mathrm{~g} \mathrm{~kg}^{-1}$ de M.O.; CTC de $47,6 \mathrm{mmol}_{\mathrm{c}} \mathrm{dm}^{-3} \mathrm{e} 4 \mathrm{mmol}_{\mathrm{c}} \mathrm{dm}^{-3} \mathrm{de} \mathrm{Al}$. O composto de lixo urbano, cuja análise química é apresentada na tabela 1 , foi aplicado duas vezes no primeiro ano (inverno e verão) e uma vez por ano nos três anos consecutivos (inverno, verão e verão), nas doses de 0 ,

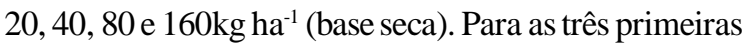
aplicações, utilizou-se o composto fornecido pelo Departamento Municipal de Limpeza Urbana da Prefeitura de Porto Alegre (RS) e, nas duas últimas, o composto da Prefeitura de Novo Hamburgo (RS).

Após o preparo do solo e controle de plantas invasoras, a área experimental foi dividida em vinte $\mathrm{e}$ quatro parcelas de $3 \times 4 \mathrm{~m}$. As parcelas foram separadas entre si por $1 \mathrm{~m}$ de largura e os blocos separados por $2 \mathrm{~m}$ de largura, perfazendo uma área total de $480 \mathrm{~m}^{2}$.

O composto foi aplicado na superfície do solo, distribuído na área total das parcelas, de acordo com os tratamentos, e incorporado com arado de disco, seguido de duas gradagens. Como comparativo, foi utilizado um tratamento com aplicação de calcário (PRNT $=102 \%$ ), na dose de 2,2t ha $\mathrm{t}^{-1}$, e de adubo mineral, de acordo com a análise do solo e a recomendação para as culturas utilizadas (COMISSÃO, 2004).

Após 30 dias da adição do composto, foi cultivado aveia (Avena strigosa), milho (Zea mays), aveia, milheto (Pennisetum americanum) e milheto,

Tabela 1 - Características químicas dos compostos de lixo urbano domiciliar, aplicados no experimento durante os quatro anos de avaliação (médias de três repetições).

\begin{tabular}{|c|c|c|c|c|}
\hline & \multicolumn{4}{|c|}{ - } \\
\hline & POA $1 \stackrel{a}{a}$ e $2^{\underline{a}} * *$ & POA 3‥ & $\mathrm{NH} 44^{\mathrm{a}} * * *$ & NH $5^{\mathrm{a}}$ \\
\hline $\mathrm{pH}\left(\mathrm{H}_{2} \mathrm{O}\right)$ & 7,3 & 7,7 & 7,5 & 7,7 \\
\hline C orgânico $\left(\mathrm{g} \mathrm{kg}^{-1}\right)$ & 80,7 & 83,9 & 68,0 & 70,0 \\
\hline $\mathrm{N}$ total $\left(\mathrm{g} \mathrm{kg}^{-1}\right)$ & 7,2 & 7,0 & 6,5 & 6,7 \\
\hline P total $\left(\mathrm{g} \mathrm{kg}^{-1}\right)$ & 2,3 & 2,3 & 2,1 & 1,9 \\
\hline $\mathrm{K}$ total $\left(\mathrm{g} \mathrm{kg}^{-1}\right)$ & 4,3 & 3,5 & 3,5 & 3,6 \\
\hline Ca total $\left(\mathrm{g} \mathrm{kg}^{-1}\right)$ & 21,5 & 30,8 & 12,4 & 13,1 \\
\hline $\mathrm{Mg}$ total $\left(\mathrm{g} \mathrm{kg}^{-1}\right)$ & 1,5 & 1,9 & 5,2 & 2,3 \\
\hline Na total $\left(\mathrm{mg} \mathrm{kg}^{-1}\right)$ & 997 & 2819 & 4224 & 1174 \\
\hline $\operatorname{RAS}^{* * * *}(\%)$ & 0,08 & 0,09 & 0,20 & 0,15 \\
\hline
\end{tabular}

* POA 1 $1^{\mathrm{a}}, 2^{\mathrm{a}}$. e $3^{\mathrm{a}}$. aplicação. Compostos fornecidos pelo Departamento Municipal de Limpeza Urbana da Prefeitura Municipal de Porto Alegre (RS); NH 4⿳亠丷⿵冂丶 e e ${ }^{a}$. aplicação. Compostos fornecidos pela Prefeitura Municipal de Novo Hamburgo (RS).

* Média dos resultados da análise dos dois compostos utilizados.

** Composto contaminado com solo.

**** Relação de adsorção de sódio. 
respectivamente, em cada uma das cinco épocas de adições. No tratamento com adubação mineral, foram aplicados $110 \mathrm{~kg} \mathrm{ha}^{-1}$ de $\mathrm{P}_{2} \mathrm{O}_{5}, 130 \mathrm{~kg} \mathrm{ha}^{-1}$ de $\mathrm{K}_{2} \mathrm{O}$ e $260 \mathrm{~kg}$ $\mathrm{ha}^{-1}$ de $\mathrm{N}$ ao longo dos dois primeiros cultivos, ou seja, no primeiro ano do experimento, na semeadura da cultura de aveia e com efeito residual para a cultura do milho. No segundo ano, a cultura da aveia foi adubada com $40 \mathrm{~kg} \mathrm{ha}^{-1}$ de $\mathrm{P}_{2} \mathrm{O}_{5}, 40 \mathrm{~kg} \mathrm{ha}^{-1}$ de $\mathrm{K}_{2} \mathrm{O}$ e $140 \mathrm{~kg} \mathrm{ha}^{-1}$ de $\mathrm{N}\left(20 \mathrm{~kg} \mathrm{ha}^{-1}\right.$ na semeadura, $70 \mathrm{~kg} \mathrm{ha}^{-1}$ no perfilhamento e $50 \mathrm{~kg} \mathrm{ha}^{-1}$ ao início do florescimento). Na cultura do milheto, cultivado consecutivamente no terceiro e quarto ano do experimento, foi aplicado $80 \mathrm{~kg} \mathrm{ha}^{-1} \mathrm{de}$ $\mathrm{P}_{2} \mathrm{O}_{5}, 40 \mathrm{~kg} \mathrm{ha}^{-1} \mathrm{de} \mathrm{K}_{2} \mathrm{O}$ e $200 \mathrm{~kg} \mathrm{ha}^{-1}$ de N $\left(20 \mathrm{~kg} \mathrm{ha}^{-1} \mathrm{na}\right.$ semeadura, $80 \mathrm{~kg} \mathrm{ha}^{-1}$ no perfilhamento e $80 \mathrm{~kg} \mathrm{ha}^{-1}$ ao início do florescimento).

As amostras de solo foram coletadas da camada superficial, a uma profundidade de até $20 \mathrm{~cm}$, secas ao ar e à sombra e então passadas por peneira com malha de $0,5 \mathrm{~mm}$. As amostragens foram feitas em setembro ( $1^{\circ}$ ano), março e novembro ( $2^{\circ}$ ano), setembro (3- ano) e fevereiro (4o ano) após a colheita das respectivas culturas. Para todas as amostras, efetuaramse as caracterizações básicas de N-total, P e K disponíveis, $\mathrm{Na}, \mathrm{Ca}, \mathrm{Mg}, \mathrm{Mn}$ e $\mathrm{Al}$ trocáveis, carbono orgânico e CTC (soma dos cátions trocáveis, excluindo $\mathrm{o}^{+}$), através da metodologia descrita por TEDESCO et al. (1995). A relação de adsorção de sódio (RAS) foi calculada a partir da regressão estabelecida por RICHARDS (1969), utilizando a razão de sódio trocável (RST), definida por RST=ST/(CTC-ST), igual a 100, em que ST representa o sódio trocável, em $\mathrm{mmol}_{\mathrm{c}} \mathrm{dm}^{-3}$.

$\mathrm{O}$ experimento foi instalado em delineamentos em blocos completamente casualizados, com quatro repetições. Os resultados foram submetidos à análise de variância e, quando ocorreram diferenças detectadas pelo teste $\mathrm{F}(\mathrm{P}<0,05)$, as médias foram comparadas utilizando-se o teste de Tukey a $5 \%$ de probabilidade. As análises estatísticas e as de correlação foram feitas utilizando-se o Pacote Estatístico SigmaStat.

\section{RESULTADOS E DISCUSSÃO}

Observou-se que o $\mathrm{pH}$ medido em água aumentou à medida que aumentaram as doses de composto (Tabela 2). Em todas as cinco avaliações

Tabela 2 - Valores de pH (em água), de alumínio trocável e de capacidade de troca catiônica (CTC) do solo, após a aplicação de composto de lixo urbano nas doses de 20,40, 80 e 160 $\mathrm{ha}^{-1}$, durante cinco aplicações sucessivas. As amostragens de solo foram realizadas logo após a coleta das culturas de cada período de aplicação do composto.

\begin{tabular}{|c|c|c|c|c|c|}
\hline Tratamentos & $1^{\mathrm{a}}$ aplicação & $2^{2}$ aplicação & $3^{\text {a }}$ aplicação & $4^{\mathrm{a}}$ - aplicação & $5^{\mathrm{a}}$ aplicação \\
\hline & & & \multicolumn{3}{|c|}{ 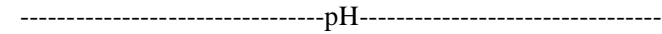 } \\
\hline Sem Composto & $4,7 c^{*}$ & $4,7 \mathrm{e}$ & $4,7 \mathrm{c}$ & $5,1 \mathrm{e}$ & $4,9 \mathrm{c}$ \\
\hline Composto $20 \mathrm{tha}^{-1}$ & $5,1 \mathrm{~b}$ & $5,1 \mathrm{~cd}$ & $5,4 \mathrm{bc}$ & $5,7 \mathrm{~d}$ & $5,9 \mathrm{~b}$ \\
\hline Composto $40 \mathrm{tha}^{-1}$ & $5,2 b$ & $5,3 \mathrm{c}$ & $5,5 \mathrm{~b}$ & $6,2 \mathrm{c}$ & $6,2 \mathrm{ab}$ \\
\hline Composto $80 \mathrm{tha}^{-1}$ & $5,8 \mathrm{a}$ & $5,8 b$ & $6,3 \mathrm{a}$ & $6,8 \mathrm{~b}$ & $6,4 \mathrm{ab}$ \\
\hline Composto $160 \mathrm{tha}^{-1}$ & $5,9 \mathrm{a}$ & $6,7 \mathrm{a}$ & $6,7 \mathrm{a}$ & $7,3 \mathrm{a}$ & $6,6^{\mathrm{a}}$ \\
\hline \multirow{2}{*}{ Calcário e NPK*** } & $5,1 \mathrm{~b}$ & $5,00 \mathrm{de}$ & $4,8 \mathrm{bc}$ & $5,2 \mathrm{e}$ & $4,8 \mathrm{c}$ \\
\hline & & & \multicolumn{3}{|c|}{ 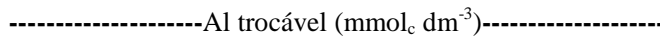 } \\
\hline Sem Composto & $4,9 \mathrm{a}$ & $5,0 \mathrm{a}$ & ND & ND & ND \\
\hline Composto 20t ha ${ }^{-1}$ & $4,4 \mathrm{ab}$ & $2,4 \mathrm{~b}$ & ND & ND & ND \\
\hline Composto $40 \mathrm{tha}^{-1}$ & $3,7 \mathrm{ab}$ & $0,9 \mathrm{c}$ & ND & ND & ND \\
\hline Composto $80 \mathrm{tha}^{-1}$ & $0,7 \mathrm{c}$ & $0,3 \mathrm{c}$ & ND & ND & ND \\
\hline Composto $160 \mathrm{tha}^{-1}$ & $0,4 \mathrm{c}$ & $0,0 \mathrm{c}$ & ND & ND & ND \\
\hline \multirow[t]{2}{*}{ Calagem e NPK } & $3,4 \mathrm{~b}$ & $2,7 \mathrm{~b}$ & ND & ND & ND \\
\hline & & & \multicolumn{3}{|c|}{-------Capacidade de troca de cát ions $\left(\mathrm{mmol}_{\mathrm{c}} \mathrm{dm}^{-3}\right)$-----. } \\
\hline Sem Composto & $\mathrm{ND}^{* *}$ & $35,9 \mathrm{c}$ & $60,0 \mathrm{c}$ & $43,0 \mathrm{e}$ & $64,0 \mathrm{~d}$ \\
\hline Composto 20t ha $\mathrm{a}^{-1}$ & ND & $43,2 \mathrm{c}$ & $67,0 \mathrm{c}$ & $64,0 \mathrm{~cd}$ & $90,0 \mathrm{bc}$ \\
\hline Composto 40t ha ${ }^{-1}$ & ND & $45,6 \mathrm{c}$ & $91,0 b$ & $75,0 \mathrm{c}$ & $109,0 \mathrm{~b}$ \\
\hline Composto $80 \mathrm{tha}^{-1}$ & ND & $63,1 b$ & $107,0 \mathrm{~b}$ & $98,0 \mathrm{~b}$ & $137,0 \mathrm{a}$ \\
\hline Composto $160 \mathrm{tha}^{-1}$ & ND & $80,8 \mathrm{a}$ & $140,0 \mathrm{a}$ & $120,0 \mathrm{a}$ & $157,0 \mathrm{a}$ \\
\hline Calagem e NPK & ND & $40,9 \mathrm{c}$ & $66,0 \mathrm{c}$ & $52,0 \mathrm{de}$ & $68,0 \mathrm{~cd}$ \\
\hline
\end{tabular}

*Médias seguidas de letras iguais não diferem estatisticamente entre tratamentos pelo teste Tukey $(\mathrm{P}<0,05)$.

$* * \mathrm{ND}=$ não determinado.

*** A aplicação de calcário foi realizada em uma aplicação $\left(2,2 \mathrm{t} \mathrm{ha}{ }^{-1}\right)$ com calcário com PRNT=102\% no primeiro ano de cultivo. Já o adubo mineral foi aplicado na base, de acordo com COMISSÃO (2004) para cada cultura.

Ciência Rural, v.41, n.3, mar, 2011. 
realizadas no período de quatro anos, verificou-se que as adições de 80 e $160 \mathrm{t} \mathrm{ha}^{-1}$ de composto resultaram em valores de $\mathrm{pH}$ superiores aos demais tratamentos. As novas adições de composto acentuaram ainda mais as diferenças no pH nos tratamentos, ou seja, à medida que novas aplicações são feitas, maiores são as diferenças entre os valores de $\mathrm{pH}$ do solo. A dose de $160 \mathrm{t} \mathrm{ha}^{-1}$ proporcionou valores de $\mathrm{pH}$ superiores aos verificados nos demais tratamentos. A dose de 40t ha-1 resultou em $\mathrm{pH}$ superior ao obtido com a aplicação de calcário, o qual não se diferenciou do obtido com $20 \mathrm{t} \mathrm{ha}^{-1}$ de composto. Evidencia-se, deste modo, o efeito corretivo do composto de lixo aplicado em solo ácido, referido na literatura com doses a partir de $20 \mathrm{t}$ ha $^{-1}$ (CAVALLET et al., 2004). O aumento no $\mathrm{pH}$ pode ser devido à maior mineralização da matéria orgânica e produção subsequente de íons $\mathrm{OH}^{-}$, bem como pela

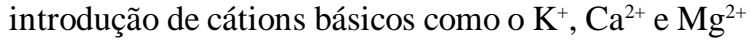
(HARGREAVES et al., 2008). Observou-se que a dose de calcário foi subestimada no tratamento com adição de calcário e adubo NPK, uma vez que não atingiu o pH 6,0 desejado.

Para o teor de alumínio trocável, verificouse uma diminuição, à medida que as doses do composto aumentaram (Tabela 2). O efeito foi mais acentuado após a segunda aplicação de composto, quando os tratamentos com doses de $20 \mathrm{tha}^{-1}$ de composto já apresentavam uma redução do Al trocável da mesma ordem que os tratamentos onde foi aplicado calcário. Além do efeito direto na redução da concentração de Al trocável, a aplicação do resíduo proporcionou também a redução da percentagem de saturação da CTC com Al através da adição de uma quantidade apreciável de outros cátions trocáveis, contribuindo para diminuir a toxidez do $\mathrm{Al}^{3+}$. Resultados semelhantes também foram observados por ABREU JUNIOR et al. (2000), que verificaram que a adição de composto de lixo promoveu o aumento do $\mathrm{pH}$ e reduziu a acidez potencial do solo, em condições de casa de vegetação.

Os valores de CTC no tratamento com aplicação de 160t ha-1 de composto de lixo, após a quinta aplicação, foi mais de duas vezes superior à média verificada no tratamento sem adição de composto, sendo que a CTC do tratamento com 80t ha ${ }^{-1}$ de composto foi em média $75 \%$ superior ao tratamento sem composto (Tabela 2). Os tratamentos com aplicações de até $40 \mathrm{t} \mathrm{ha}^{-1}$ de composto não apresentaram diferenças significativas com o tratamento sem composto, embora tenha sido observado um aumento em função das quantidades aplicadas. Em solos ácidos, como o do presente experimento, ABREU JUNIOR et al. (2001) verificaram um aumento de $42 \%$ na CTC, quando adicionado $60 \mathrm{t} \mathrm{ha}^{-1} \mathrm{de}$ composto de lixo proveniente da cidade de São Paulo. Esses autores atribuíram o aumento da CTC ao acréscimo de cargas negativas provenientes da matéria orgânica contida no composto. ALMEIDA (2003) verificou que o composto aumentou a CTC de substratos utilizados para o cultivo de mudas de maracujazeiro.

Foi constatado um aumento progressivo no teor de carbono orgânico do solo com as doses de composto de lixo, com a estabilização dos valores no decorrer dos quatro anos de avaliação (Tabela 3). A avaliação realizada logo após a primeira aplicação revelou um efeito significativo do composto sobre o teor de carbono, a partir da dose de $80 \mathrm{tha}^{-1}$. O mesmo comportamento foi observado logo após a segunda aplicação, mas não apresentou efeito acumulado das duas aplicações de composto, em relação à primeira aplicação. Possivelmente, a velocidade da decomposição microbiana do composto orgânico variou em função da resistência dos constituintes orgânicos presentes no material, bem como de fatores do meio que condicionam a atividade microbiana (WEI et al., 2007).

O teor de $\mathrm{N}$ total do solo foi aumentado de acordo com o aumento da dose de composto, sendo as duas maiores doses de composto os tratamentos com maior teor de $\mathrm{N}$ total obtido (Tabela 3 ). Como resultado da segunda adição de composto, as diferenças se acentuaram, sendo que, na dose de $160 \mathrm{t} \mathrm{ha}^{-1}$, o teor de $\mathrm{N}$ elevou-se em $1,8 \mathrm{~g} \mathrm{~kg}^{-1}$, superiores aos $1,5 \mathrm{~g} \mathrm{~kg}^{-1}$ obtidos na dose de $80 \mathrm{t} \mathrm{ha}^{-1}$. Isso ocorreu devido aos teores relativamente baixos de $\mathrm{N}$ verificados no composto. Entretanto, observou-se uma alta relação entre os teores, no solo, de $\mathrm{N}$ total e o de carbono orgânico, indicando que o composto pode ser utilizado como um fornecedor de $\mathrm{N}$ de liberação lenta e dependente da atividade microbiana, conforme observado anteriormente nos trabalhos de OLIVEIRA et al. (2001) e CORDOVIL et al. (2007).

O teor de fósforo extraível no solo foi maior nos tratamentos com as doses maiores de composto. Os teores observados quando se adicionou 20 e $40 \mathrm{t}$ $\mathrm{ha}^{-1}$, bem como quando adicionou-se fertilizante mineral, não foram diferentes do encontrado no tratamento sem composto (Tabela 3). À medida que o composto de lixo foi reaplicado, verificou-se de maneira concomitante um aumento nos teores de $\mathrm{P}$, fato este já esperado, uma vez que a aplicação de resíduos orgânicos geralmente eleva o teor de $\mathrm{P}$ disponível no solo, normalmente a níveis acima dos obtidos com a aplicação da mesma quantidade de $\mathrm{P}$ na forma de superfosfato (RUPPENTHAL \& CASTRO, 2005). O aumento do P disponível no solo após a adição de material orgânico 
Tabela 3 - Valores de carbono orgânico, do nitrogênio total, do fósforo e do potássio extraíveis no solo após a aplicação de composto de lixo urbano nas doses de 20,40, 80 e $160 \mathrm{t} \mathrm{ha}^{-1}$, durante cinco aplicações sucessivas. As amostragens de solo foram realizadas logo após a coleta das culturas de cada período de aplicação do composto.

\begin{tabular}{|c|c|c|c|c|c|}
\hline Tratamentos & $1^{\mathrm{a}}$ aplicação & $2^{\mathrm{a}}$ aplicação & $3^{\mathrm{a}}$ aplicação & $4^{\mathrm{a}}$ aplicação & $5^{\mathrm{a}}$ aplicação \\
\hline & & & \multicolumn{3}{|c|}{ - } \\
\hline Sem Composto & $13,3 \mathrm{c}^{*}$ & $13,0 \mathrm{c}$ & $14,5 \mathrm{c}$ & $12,8 \mathrm{c}$ & $15,1 \mathrm{~d}$ \\
\hline Composto $20 \mathrm{tha}^{-1}$ & $13,3 \mathrm{c}$ & $13,3 \mathrm{c}$ & $15,7 \mathrm{c}$ & $14,5 \mathrm{c}$ & $17,4 \mathrm{~cd}$ \\
\hline Composto $40 \mathrm{tha}^{-1}$ & $13,4 \mathrm{c}$ & $13,3 \mathrm{c}$ & $14,4 \mathrm{c}$ & $15,7 \mathrm{bc}$ & $19,8 \mathrm{c}$ \\
\hline Composto 80t ha ${ }^{-1}$ & $14,4 \mathrm{~b}$ & $15,1 \mathrm{~b}$ & $21,5 b$ & $18,6 b$ & $25,0 \mathrm{~b}$ \\
\hline Composto $160 \mathrm{tha}^{-1}$ & $16,2 \mathrm{a}$ & $19,3 \mathrm{a}$ & $29,7 \mathrm{a}$ & $27,3 \mathrm{a}$ & $28,5 \mathrm{a}$ \\
\hline \multirow[t]{2}{*}{ Calagem e NPK $* * *$} & $12,7 \mathrm{c}$ & $13,3 \mathrm{c}$ & $16,9 \mathrm{c}$ & $14,0 \mathrm{c}$ & $15,7 \mathrm{~d}$ \\
\hline & & & \multicolumn{3}{|c|}{ - } \\
\hline Sem Composto & $1,2 \mathrm{c}$ & $1,1 d$ & $\mathrm{ND} * *$ & ND & ND \\
\hline Composto $20 \mathrm{tha}^{-1}$ & $1,3 \mathrm{bc}$ & $1,2 \mathrm{~cd}$ & ND & ND & ND \\
\hline Composto $40 \mathrm{tha}^{-1}$ & $1,4 \mathrm{~b}$ & $1,2 \mathrm{~cd}$ & ND & ND & ND \\
\hline Composto $80 \mathrm{tha}^{-1}$ & $1,6 \mathrm{a}$ & $0,15 b$ & ND & ND & ND \\
\hline Composto $160 \mathrm{tha}^{-1}$ & $1,7 \mathrm{a}$ & $1,8 \mathrm{a}$ & ND & ND & ND \\
\hline \multirow[t]{2}{*}{ Calagem e NPK } & $1,3 \mathrm{bc}$ & $1,3 \mathrm{c}$ & ND & ND & ND \\
\hline & & & \multicolumn{3}{|c|}{ - Pextraível $\left(\mathrm{mg} \mathrm{kg}^{-1}\right)$} \\
\hline Sem Composto & $14 \mathrm{c}$ & $7 \mathrm{c}$ & $7 \mathrm{c}$ & $6 \mathrm{c}$ & $2 d$ \\
\hline Composto $20 \mathrm{tha}^{-1}$ & $16 \mathrm{c}$ & $13 \mathrm{c}$ & $11 \mathrm{c}$ & $13 \mathrm{c}$ & $20 \mathrm{~cd}$ \\
\hline Composto $40 \mathrm{tha}^{-1}$ & $17 \mathrm{bc}$ & $19 \mathrm{bc}$ & $21 b$ & $21 \mathrm{c}$ & $49 \mathrm{c}$ \\
\hline Composto $80 \mathrm{tha}^{-1}$ & $23 \mathrm{ab}$ & $43 b$ & $44 \mathrm{a}$ & $49 \mathrm{~b}$ & $88 \mathrm{~b}$ \\
\hline Composto $160 \mathrm{tha}^{-1}$ & $28 \mathrm{a}$ & $98 \mathrm{a}$ & $79 \mathrm{c}$ & $86 \mathrm{a}$ & $130 \mathrm{a}$ \\
\hline \multirow[t]{2}{*}{ Calagem e NPK } & $14 \mathrm{c}$ & $11 \mathrm{c}$ & $7 \mathrm{c}$ & $7 \mathrm{c}$ & $9 \mathrm{~d}$ \\
\hline & & & \multicolumn{3}{|c|}{ - K extraível $\left(\mathrm{mg} \mathrm{kg}^{-1}\right)$ - } \\
\hline Sem Composto & $175 \mathrm{a}$ & $150 \mathrm{a}$ & 130d & $118 \mathrm{c}$ & $170 \mathrm{bcd}$ \\
\hline Composto $20 \mathrm{tha}^{-1}$ & $176 a$ & $158 \mathrm{a}$ & $127 \mathrm{~d}$ & $127 b c$ & $157 \mathrm{~cd}$ \\
\hline Composto $40 \mathrm{tha}^{-1}$ & $183 a$ & $159 a$ & $158 \mathrm{c}$ & $146 b$ & $216 a b c$ \\
\hline Composto $80 \mathrm{tha}^{-1}$ & $210 \mathrm{a}$ & $180 \mathrm{a}$ & $191 b$ & $181 \mathrm{a}$ & $239 \mathrm{ab}$ \\
\hline Composto $160 \mathrm{tha}^{-1}$ & $215 \mathrm{a}$ & $182 \mathrm{a}$ & $231 \mathrm{a}$ & $198 \mathrm{a}$ & $286 a$ \\
\hline Calagem e NPK & $140 \mathrm{a}$ & $153 \mathrm{a}$ & $136 \mathrm{~cd}$ & $124 b c$ & $134 d$ \\
\hline
\end{tabular}

*Médias seguidas de letras iguais não diferem estatisticamente entre tratamentos pelo teste Tukey $(\mathrm{P}<0,05)$.

**ND = não determinado

*** A aplicação de calcário foi realizada em 1 aplicação $\left(2,2 \mathrm{t}\right.$ ha $\left.^{-1}\right)$ com calcário com PRNT=102\% no primeiro ano de cultivo. Já o adubo mineral foi aplicado na base, de acordo com COMISSÃO (2004) para cada cultura.

está associado à mineralização do $\mathrm{P}$ orgânico e à redução da fixação do $\mathrm{P}$ na fração mineral, promovida pela competição dos ânions orgânicos pelos sítios de adsorção na superfície dos oxi-hidróxidos de $\mathrm{Fe}$ e $\mathrm{Al}$ (CAVALLET et al., 2004).

O teor de potássio no solo não foi alterado entre os tratamentos ao final da segunda aplicação, apesar de observar-se um aumento com as doses (Tabela 3). A ausência de resposta do $\mathrm{K}$ se deve provavelmente ao conteúdo relativamente baixo deste elemento no composto. Observou-se um aumento nos teores de K, à medida que foram feitas novas aplicações, sendo este mais pronunciado nas três maiores doses.

Os teores de $\mathrm{Ca}$ e $\mathrm{Mg}$ do solo, extraíveis com $\mathrm{KCl}$, aumentaram proporcionalmente às doses do composto (Tabela 4). Observaram-se ainda aumentos nos teores desses elementos à medida que o composto foi aplicado sucessivamente. Em relação ao sódio, os teores no solo aumentaram em função das doses de composto, sendo que as doses mais elevadas resultaram, na primeira avaliação, em teores médios iguais ao dobro do verificado nos demais tratamentos (Tabela 4). Na segunda avaliação, foram observados teores de $\mathrm{Na}$ inferiores aos verificados anteriormente, a exceção da dose de 160 t ha $^{-1}$, indicando uma provável lixiviação deste elemento no decorrer do experimento. Apesar do aumento do teor de $\mathrm{Na}$ e do aumento da relação de adsorção de sódio, os valores obtidos desta relação para as maiores doses aplicadas (Tabela 4) não indicam uma concentração de sódio a ponto de prejudicar o desenvolvimento das culturas ou de comprometer a estrutura do solo. Tem sido sugerido 
Tabela 4 - Valores de cálcio, magnésio e sódio trocáveis e da razão de adsorção de sódio (RAS) no solo após a aplicação de composto de lixo urbano nas doses de 20,40, 80 e $160 \mathrm{t} \mathrm{ha}^{-1}$, durante de cinco aplicações sucessivas. As amostragens de solo foram realizadas logo após a coleta das culturas de cada período de aplicação do composto.

\begin{tabular}{|c|c|c|c|c|c|}
\hline Tratamentos & $1^{\text {a }}$ aplicação & $2^{\underline{a}}$ aplicação & $3^{\mathrm{a}}$ aplicação & $4^{\mathrm{a}}$ aplicação & $5^{\mathrm{a}}$ aplicação \\
\hline & & & \multicolumn{3}{|c|}{ - Ca trocável $\left(\mathrm{mmol}_{\mathrm{c}} \mathrm{dm}^{-3}\right)$} \\
\hline Sem Composto & $19 \mathrm{~d}^{*}$ & $18 \mathrm{~d}$ & $22 \mathrm{c}$ & $19 \mathrm{~d}$ & $25 \mathrm{e}$ \\
\hline Composto $20 \mathrm{tha}^{-1}$ & $22 \mathrm{~cd}$ & $27 \mathrm{~cd}$ & $37 \mathrm{c}$ & $42 \mathrm{c}$ & $62 \mathrm{~d}$ \\
\hline Composto $40 \mathrm{t} \mathrm{ha}^{-1}$ & $24 \mathrm{c}$ & $30 \mathrm{c}$ & $57 \mathrm{~b}$ & $52 \mathrm{c}$ & $80 \mathrm{c}$ \\
\hline Composto 80t ha ${ }^{-1}$ & $32 b$ & $44 \mathrm{~b}$ & $71 \mathrm{~b}$ & $74 \mathrm{~b}$ & $107 \mathrm{~b}$ \\
\hline Composto $160 \mathrm{tha}^{-1}$ & $38 \mathrm{a}$ & $60 \mathrm{a}$ & $100 \mathrm{a}$ & $94^{\mathrm{a}}$ & $123 \mathrm{a}$ \\
\hline \multirow[t]{2}{*}{ Calagem e NPK *** } & $24 \mathrm{c}$ & $24 \mathrm{~cd}$ & $29 \mathrm{c}$ & $27 \mathrm{~d}$ & $31 \mathrm{e}$ \\
\hline & & & \multicolumn{3}{|c|}{ - } \\
\hline Sem Composto & $8 \mathrm{~d}$ & $7 \mathrm{~b}$ & $14 \mathrm{~d}$ & $6 \mathrm{~d}$ & $10 \mathrm{~d}$ \\
\hline Composto $20 \mathrm{tha}^{-1}$ & $9 \mathrm{~cd}$ & $9 \mathrm{~b}$ & $15 \mathrm{~cd}$ & $9 \mathrm{c}$ & $14 \mathrm{~cd}$ \\
\hline Composto $40 \mathrm{tha}^{-1}$ & $10 \mathrm{bcd}$ & $9 \mathrm{~b}$ & $20 \mathrm{bc}$ & $11 \mathrm{bc}$ & $16 \mathrm{c}$ \\
\hline Composto $80 \mathrm{tha}^{-1}$ & $11 \mathrm{ab}$ & $2 \mathrm{a}$ & $24 \mathrm{~b}$ & $13 \mathrm{ab}$ & $19 \mathrm{~b}$ \\
\hline Composto $160 \mathrm{tha}^{-1}$ & $12 \mathrm{a}$ & $3 a$ & $29 \mathrm{a}$ & $16 \mathrm{a}$ & $21 \mathrm{a}$ \\
\hline \multirow{2}{*}{ Calagem e NPK } & $10 \mathrm{bc}$ & $9 b$ & $16 \mathrm{~cd}$ & $9 \mathrm{~cd}$ & $10 \mathrm{~cd}$ \\
\hline & & & \multicolumn{3}{|c|}{ 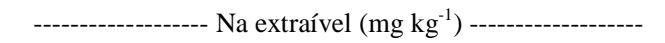 } \\
\hline Sem Composto & $12 \mathrm{c}$ & $8 \mathrm{c}$ & $12 \mathrm{c}$ & $4 \mathrm{~d}$ & $7 \mathrm{c}$ \\
\hline Composto $20 \mathrm{tha}^{-1}$ & $17 \mathrm{c}$ & $11 \mathrm{c}$ & $21 \mathrm{c}$ & $12 \mathrm{~cd}$ & $22 \mathrm{bc}$ \\
\hline Composto $40 \mathrm{tha}^{-1}$ & $18 \mathrm{bc}$ & $14 \mathrm{c}$ & $29 \mathrm{bc}$ & $17 \mathrm{c}$ & $37 \mathrm{c}$ \\
\hline Composto $80 \mathrm{tha}^{-1}$ & $32 \mathrm{ab}$ & $26 \mathrm{~b}$ & $42 b$ & $34 \mathrm{~b}$ & $66 b$ \\
\hline Composto $160 \mathrm{tha}^{-1}$ & $39 \mathrm{a}$ & $62 \mathrm{a}$ & $71 \mathrm{a}$ & $59 \mathrm{a}$ & $106 \mathrm{a}$ \\
\hline \multirow[t]{2}{*}{ Calagem e NPK } & $14 \mathrm{c}$ & $10 \mathrm{c}$ & $12 \mathrm{c}$ & $4 \mathrm{~d}$ & $8 \mathrm{c}$ \\
\hline & $\mathrm{ND}^{* *}$ & $0,15 \mathrm{c}$ & $0,12 \mathrm{c}$ & $\begin{array}{c}\text { RAS (\%)-- } \\
0,05 \mathrm{~d}\end{array}$ & $0,07 \mathrm{c}$ \\
\hline Composto $20 \mathrm{tha}^{-1}$ & ND & $0,16 b c$ & $0,18 b c$ & $0,10 \mathrm{~cd}$ & $0,15 b c$ \\
\hline Composto $40 \mathrm{tha}^{-1}$ & ND & $0,18 b c$ & $0,20 b c$ & $0,13 \mathrm{c}$ & $0,23 b c$ \\
\hline Composto $80 \mathrm{tha}^{-1}$ & ND & $0,21 b$ & $0,26 \mathrm{~b}$ & $0,22 \mathrm{c}$ & $0,36 \mathrm{ab}$ \\
\hline Composto $160 \mathrm{tha}^{-1}$ & ND & $0,32 \mathrm{a}$ & $0,38^{\mathrm{a}}$ & $0,35 \mathrm{a}$ & $0,54 \mathrm{a}$ \\
\hline Calagem e NPK & ND & $0,16 b c$ & $0,11 \mathrm{c}$ & $0,04 d$ & $0,08 \mathrm{c}$ \\
\hline
\end{tabular}

*Médias seguidas de letras iguais não diferem estatisticamente entre tratamentos pelo teste Tukey $(\mathrm{P}<0,05)$.

**ND = não determinado.

*** A aplicação de calcário foi realizada em 1 aplicação $\left(2,2 \mathrm{t}\right.$ ha $\left.{ }^{-1}\right)$ com calcário com PRNT=102\% no primeiro ano de cultivo. Já o adubo mineral foi aplicado na base, de acordo com COMISS ÃO (2004) para cada cultura.

que somente valores de RAS acima de 5\% é que podem causar degradação da estrutura do solo e valores acima de $10 \%$ são necessários para um desenvolvimento vegetal inadequado (BARBOSA et al., 2005). Os dados sugerem, entretanto, que a aplicação de doses mais elevadas do composto pode aumentar excessivamente a concentração do elemento, especialmente se utilizado em regiões áridas ou semi-áridas (ABREU JUNIOR et al., 2000).

\section{CONCLUSÃO}

A aplicação sucessiva de composto de lixo urbano aumenta os valores de $\mathrm{pH}$, da CTC, da relação de adsorção de sódio e os teores de $\mathrm{C}$ orgânico, de N total, de P, de Na extraível e os de Ca e o Mg trocáveis, não altera o teor de $\mathrm{K}$ extraível e diminui o teor de $\mathrm{Al}$ trocável no solo.

Pode-se inferir que aplicações anuais de composto de lixo urbano de até $80 \mathrm{t} \mathrm{ha}^{-1}$ podem ser consideradas como adequadas para melhorar ou manter as propriedades químicas do solo.

\section{REFERÊNCIAS}

ABREU JUNIOR, C.H. et al. Condutividade elétrica, reação do solo e acidez potencial em solos adubados com composto de lixo. Revista Brasileira de Ciência do Solo, Viçosa, v.24, p.635-647, 2000.

ABREU JUNIOR, C.H. et al. Cátions trocáveis, capacidade de troca de cátions e saturação por bases em solos brasileiros adubados com composto de lixo urbano. Scientia Agrícola, Piracicaba, v.58, p.813-824, 2001. Disponível em: <http://www.google.com.br/ 
$\# \mathrm{hl}=\mathrm{pt}-\mathrm{BR} \& \mathrm{biw}=1362 \& \mathrm{bih}=640 \& \mathrm{rlz}=1 \mathrm{~W} 1 \mathrm{GGLR} \_\mathrm{pt}-$ $\mathrm{BR} \& \mathrm{q}=\mathrm{ABREU}+\mathrm{JUNIOR} \% 2 \mathrm{C}+\mathrm{C} . \mathrm{H} .+\mathrm{et}+\mathrm{al} .++\mathrm{C} \% \mathrm{C} 3 \% \mathrm{~A} 1$ tions+troc\% $\mathrm{C} 3 \% \mathrm{~A} 1$ veis $\% 2 \mathrm{C}+$ capacidade+de+troca+de+c $\% \mathrm{C} 3 \% \mathrm{~A} 1$ tions $+\mathrm{e}+$ satu ra\% $3 \% \mathrm{~A} 7 \% \mathrm{C} 3 \% \mathrm{~A} 30+$ por+bases+em+solos+brasileiros+adubados+ com+composto+de+lixo+urbano.+Scientia+Agr\%C3\%ADcola\%2C+Pi racicaba\% $2 \mathrm{C}+\mathrm{v} .58 \% 2 \mathrm{C}+\mathrm{p} .813824 \% 2 \mathrm{C}+2001 . \& \mathrm{aq}=\mathrm{f} \& \mathrm{aq} \mathrm{i}=\& \mathrm{aq} \mathrm{l}=\& \mathrm{oq}=\& \mathrm{gs} \_\mathrm{r}$ fai $=\& f p=e b a d a 4146 f 3 e c c 2 e>$. Acesso em: 25 nov. 2007.

ALMEIDA, A. Composto de lixo urbano na composição química do solo e seus efeitos no desenvolvimento de mudas de maracujazeiro amarelo (Passiflora edulis f. flavicarpa L.). Revista de Biociência, Taubaté, v.9, p.7-15, 2003. Disponível em: <http://periodicos.unitau.br/ojs-2.2/index.php/biociencias/ article/view/106/81>. Acesso em: 20 nov. 2007.

BARBOSA, S.C.S. et al. Crescimento e absorção de nutrientes em bananeira irrigada com águas salinas. Revista Brasileira de Engenharia Agrícola e Ambiental, Campina Grande, v.9, supl, p.343-346, 2005. Disponível em: <http:// www.agriambi.com.br/ revista/suplemento/../343.pdf?>. Acesso em: 20 nov. 2007.

CAVALLET, L.E. et al. Aplicação de composto de lixo urbano em Latossolo Vermelho eutroférrico-alterações da percentagem de agregados e níveis de fósforo, potássio, carbono orgânico. Scientia Agraria Paranaensis, Cascavel, v.2, p.7-13, 2004. Disponível em: <http://e-revista.unioeste.br/index.php/scientiaagraria/issue/ view/57/showToc>. Acesso em: 20 out. 2007.

COMISSÃO DE FERTILIDADE DO SOLO - RS/SC. Manual de adubação e de calagem para os estados do Rio Grande do Sul e Santa Catarina. Porto Alegre: SBCS/NRS, 2004. $400 \mathrm{p}$.

CORDOVIL, C.M.S. et al. Potential mineralization of nitrogen from organic wastes to ryegrass and wheat crops. Bioresource Technology, New York, v.98, p.3265-3268, 2007. Disponível em: $<\mathrm{h} \mathrm{t} \mathrm{t} \mathrm{p} \mathrm{:} \mathrm{/} \mathrm{/} \mathrm{w} \mathrm{w} \mathrm{w} \mathrm{.} \mathrm{s} \mathrm{c} \mathrm{i} \mathrm{e} \mathrm{n} \mathrm{c} \mathrm{e} \mathrm{d} \mathrm{i} \mathrm{r} \mathrm{e} \mathrm{c} \mathrm{t} \mathrm{.} \mathrm{c} \mathrm{o} \mathrm{m/}$ science?_ob=ArticleListURL\&_method=list\&_ArticleListID=1603752846\& st $=13 \&$ view $=c \& \_a c c t=C 000037798 \& \_v e r s i o n=1 \& \_u r l V e r s i o n=0 \& \_u s e$ rid $=687304 \& \mathrm{md} 5=771 \mathrm{~d} 28 \mathrm{f} 1 \mathrm{ffl} 2 \mathrm{~b} 57 \mathrm{f} 34 \mathrm{~d} 5943 \mathrm{c} 2$ fbe6c06\&searchtype $=\mathrm{a}>$. Acesso em: 30 nov. 2007. doi:10.1016/j.biortech.2006.07.014.

ESCOSTEGUY, P.A.V. et al. Bactérias enteropatogênicas em composto de lixo domiciliar, solo e planta. Revista Brasileira de Ciência do Solo, Campinas, v.17, p.365-370, 1993.
HARGREAVES, J.C. et al. A review of the use of composted municipal solid waste in agriculture. Agriculture Ecosystems \& Environment, Berlin, v.123, p.1-14, 2008. Disponível em: <http://www.sciencedirect.com/ science? ob=ArticleListURL\& method=list\& ArticleListID $=16037548$ $03 \& \_s o r t=r \& \_s t=13 \& v i e w=c \& \_a c c t=C 000037798 \& \_v e r s i o n=1 \& \_u r l V e r s$ ion $=0 \&$ \&userid $=687304 \& \mathrm{md} 5=\mathrm{fffb} 7682 \mathrm{e} 6 \mathrm{eae} 4519116 \mathrm{a} 68 \mathrm{f} 1$ 09a6ea9\&searchtype=a>. Acesso em: 25 nov. 2008. doi:10.1016/ j.agee.2007.07.004.

MANTOVANI, J.R. et al. Alterações nos atributos de fertilidade em solo adubado com composto de lixo urbano. Revista Brasileira de Ciência do Solo, Viçosa, v.29, p.817-824, 2005. Disponível em: <http://www.scielo.br/scielo.php?script=sci_pdf\&pid=S0100$06832005000500017 \& \operatorname{lng}=\mathrm{en} \& \mathrm{nrm}=\mathrm{iso} \& \operatorname{tlng}=\mathrm{pt}>$. Acesso em: 20 nov. 2007.

OLIVEIRA, F.C. et al. Percolação de nitrato em Latossolo amarelo distrófico afetada pela aplicação de composto de lixo urbano e adubação mineral. Revista Brasileira de Ciência do Solo, Viçosa, v.25, p.731-741, 2001.

RICHARDS, L.A. Diagnosis and improvement of saline and alkali soils. Washington: USDA, 1969. 160p. (Agriculture Handbook n.60).

RUPPENTHAL, V.; CASTRO, A.M.C. Efeito do composto de lixo urbano na nutrição e produção de gladíolo. Revista Brasileira de Ciência do Solo, Viçosa, v.29, p.145-150, 2005. Disponível em: <http://www.scielo.br/scielo.php?script=sci_pdf\&pid=S0100$06832005000100016 \& \operatorname{lng}=$ en\&nrm=iso\&tlng=pt $>$. Acesso em: 20 nov. 2007.

TEDESCO, M.J. et al. Análise de solos, plantas e outros materiais. Porto Alegre: Departamento de Solos da UFRGS, 1995. 188p. (Boletim Técnico, 5).

WEI, Z. et al. Effect of inoculating microbes in municipal solid waste composting on characteristics of humic acid. Chemosphere, Amsterdam, v.68, p.368-374, 2007. Disponível em: <http:// w w w . s c i e $n$ c e d i r e c t . c o m / science?_ob=ArticleListURL\&_method=list\&_ArticleListID $=160$ $3768021 \&$ st $=13 \&$ view $=c \&$ acct $=C 000037798 \&$ \& version $=1 \&$ _ urlVersion $=0 \& \_$userid $=687304 \& \mathrm{md} 5=2713 \mathrm{c} 37 \mathrm{~b} 367 \mathrm{c} 8 \mathrm{dd} 1246 \mathrm{fb}$ 89bc489f512\&searchtype=a>. Acesso em: 15 out. 2007. doi:10.1016/j.chemosphere.2006.12.067. 